All course materials,

including the original lecture

are available as webcasts/

podcasts at

www.ers-education.org/

pages/default.aspx?id=405

\section{S. Radic ${ }^{1}$}

E. Salonini ${ }^{2}$

${ }^{1}$ Childrens Hospital for Respiratory Diseases and TB, Belgrade, Serbia. ${ }^{2}$ Azienda Ospedaliera Universitaria Integrata, Verona, Italy

\section{Correspondence \\ S. Radic \\ Stojana Protica 34 \\ 1000 Belgrade \\ Serbia \\ drugari@hotmail.com}

Elena Salonini

Centro Fibrosi Cistica

Azienda Ospedaliera

Universitaria Integrata

Piazzale Stefani, 1

37126 Verona,

Italy

elena.salonini@ospedaleuni-

verona.it

\section{Provenance}

Commissioned opinion article, not peer reviewed.

\section{Competing interests}

None declared.

\title{
Medical aerosols: ins and outs of inhalation therapy
}

\section{Summary}

The "Medical aerosols: ins and outs of inhalation therapy" ERS School Course was held in Amsterdam, the Netherlands in November 2009. The course aimed to provide state-of-the-art knowledge in understanding basic aerosol science, key issues of treating diseased airways, patient-related factors influencing efficacy of aerosol therapy and specific pharmacokinetic/ dynamic issues related to aerosol medicine. The course also emphasised regulatory issues and important factors on how to instruct and monitor the technical skills of patients.

ERS School Courses are aimed at all health professionals within the respiratory field and provide participants with high-quality information and hands-on session, leading to improvements in the daily clinical practice of all attendants. An important aspect of School Courses is the chance for delegates to meet and discuss their current clinical problems with experts in the field and also network with other delegates and make new contacts from around the world. This review gives the perspectives of two delegates from the course working in the field of aerosol medicine.

\section{Perspective from Snezana Radic}

The ERS School Course "Medical aerosols: ins and outs of inhalation therapy" was held on November 2009 in Amsterdam, the Netherlands. The 3-day course was attended by 68 healthcare professionals, including paediatricians, nurses, pulmonologists and pharmacists. The aim of the course was to provide basic knowledge regarding the principles, advantages and challenges of this route of medical drug administration, and to give an insight into the many factors that affect the delivery of aerosolised medication to the human lung, such as aerosol particle size and density. The course also covered specific inhalation techniques and the severity of lung disease. Each day comprised a beneficial mixture of theoretical lectures and practical interactive group workshops led by a strongly motivated faculty of experts in the field.

Many medical drugs are administered to the airways using aerosols and many devices for delivering aerosolised medications are now available. This route of drug delivery is noninvasive, fast acting ( $\beta_{2}$-adrenergic agonists), requires low doses, has minimal sideeffects and is painless. These advantages are priceless for both doctors and patients. The number of respiratory and nonrespiratory diseases compatible with this route of drug delivery is increasing. Aerosolised drugs with systemic effects (insulin) are already in use in the USA; however, because of cost and safety issues some products have been removed from the market. Clinical trials of an aerosolised vaccine for measles started 10 yrs ago in Mexico, and it proved to be very effective in school-aged children. The World Health Organization has decided to explore the possibility of mass immunisation for measles in clinical trials in India. An influenza vaccine administered as a nasal spray has already been approved for use.

Because different devices for delivering medical aerosols are available in different countries, delegates were introduced to and became not only familiar with, but well trained in, all three currently used types of devices (nebulisers, pressured 
metered dose inhalers (pMDIs) with or without spacers and dry powder inhalers (DPIs)) during daily hands-on sessions. Delegates also became aware that selecting the ideal aerosol delivery device for each patient is not always an easy task. It depends on the aerosol and deposition characteristics of the delivery system, availability of the drugs in the different devices, advantages and disadvantages of different devices and spacers, and on the ability and skills of patients of all age groups to inhale aerosols properly.

European Respiratory Society (ERS) guidelines on the use of nebulisers [1] were introduced as a strategy to ease the switch from older, less efficient to more efficient, new nebuliser technologies with enhanced patient safety and drug efficiency. The delegates were given algorithms to help them choose the right aerosol delivery device for children and adults. Deposition in the lungs differs according to the health of the airways, inhalation technique and fine particle dose. Physicochemical drug properties dictate the speed and duration of action, and also suggest the likelihood of systemic effects.

It is essential for healthcare professionals to remember that the major cause of "difficult asthma" is poor regimen or device compliance. The correct device is one that the patient can and will use, and the correct dose is the lowest dose that works. The device and dose is influenced by the competence and personal preference of the patient. The importance of continuing the education of the patient and healthcare educators, in addition to doctors and nurses, was strictly underlined during the course.

Creative workshops included practical measurement of aerosol particles, issues with the selection of different devices and spacers, nebuliser therapy in hospitals and the art of instruction, all guided and assisted by inspired faculty members. Case discussion sessions for adult and paediatric problems took place on the last day, giving healthcare professionals the opportunity to discuss difficult cases and challenge acquired knowledge.

Finally, I would like to thank all the organisers and faculty members for this beneficial, expertly organised meeting that will certainly result in good everyday practice by all participants.

\section{Perspective from Elena Salonini}

Medical aerosols currently play a primary role in the treatment of specific and often complex diseases requiring the delivery of drugs to the airways as well as the systemic circulation. The 3-day ERS School Course provided an all-round overview of medical aerosols using the knowledge of experts in the field. The format comprised a mixture of theoretical sessions and practical workshops promoting open participant discussion.

The first day started with a description of how aerosols interact with the human respiratory tract. The challenges of aerosol drug delivery are to optimise the most important determinants of depositions (aerosol particle size and density), to increase the efficiency of aerosol delivery by breathing through the mouth, which enhances drug deposition in the lungs, and to ensure specific techniques for the available devices. Another key point is the deposition of drugs within the lungs, which is reduced in patients with airway obstruction. The knowledge of how different factors affect drug deposition in the lungs is crucial for correct drug and device selection in clinical practice.

Different devices are available for delivering aerosols to the airways (nebulisers, pMDIs and DPIs) and therefore it is not easy to choose the right aerosol delivery device. The choice depends on the availability of specific drugs, the different characteristics and advantages/disadvantages of aerosol delivery and the skills of patients in different age groups. Once the inhalation device is chosen, a correct inhalation technique must be established. Studies have shown that inhalation technique is one of the primary factors for lung deposition. Therefore the task of the healthcare professional is to teach, guide, control and check correct use of the patient's medication.

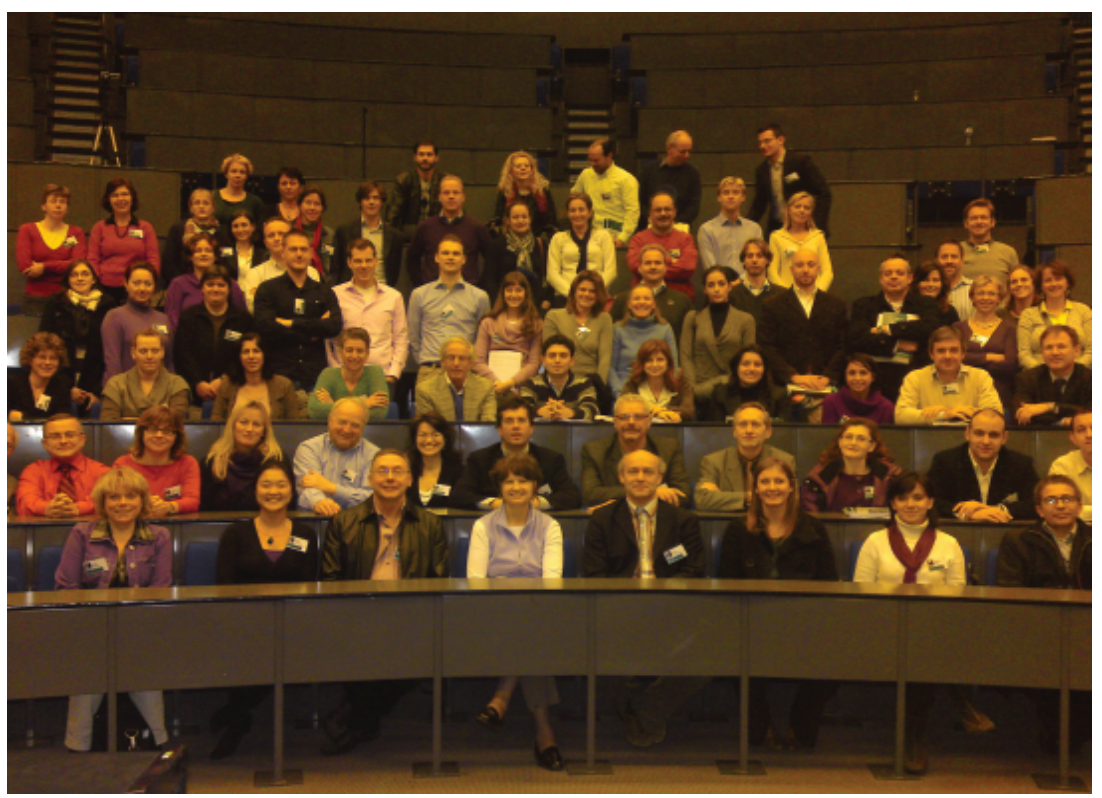




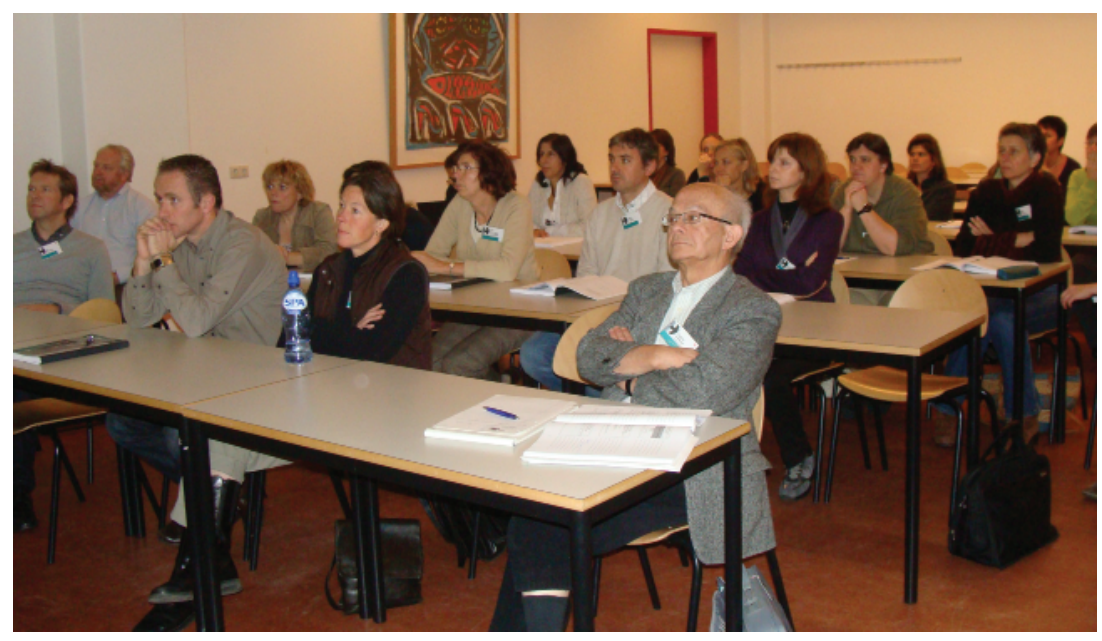

The afternoon session on the first day consisted of workshops aimed at describing the different types of pMDI, DPI and nebuliser and how to choose the right aerosol device for an adult and a child based on inhalation characteristics and potential pitfalls. I found the workshop on the measurement of aerosol particles by means of an aerodynamic particle sizer and impactor-inlet providing good quality measurements of size distributions and mass median aerodynamic diameter of inhaled aerosols of particular interest. The participants could directly appreciate the different aerosol size distribution and the dosage delivered by pMDI (with and without spacers, both plastic and anti-static), breath-actuated pMDI and DPI during this practical session.

The second day began with a series of lectures explaining the fate of an inhaled dose and the rationale of aerosol therapy in asthma, chronic obstructive pulmonary disease and cystic fibrosis. Drug deposition properties depend on particle size, airway health and inhalation technique whereas the onset and duration of action is determined by chemical properties (e.g. water solubility). Furthermore it is essential to know what clinical effects can be expected by inhaling different drugs and the correct inhaler should also be determined by individuals needs because the right device "is one that the patient can and will use correctly" (true compliance).

The afternoon session included essential clinical information, such as the easiest way to teach a procedure to the patients, which is the "DDD" sequence (demonstrate, discuss and do (together and then alone)). The practical hands-on session with new nebuliser technology was very useful as the participants could try out the new aerosolgeneration technologies (vibrating mesh technology and soft-mist inhaler) and nebulisers that are breathing pattern-dependent. The ERS nebuliser guidelines provide a strategy for safer and more effective use of the new nebulisers in clinical practice [1].

The final day included lectures focused on the role of healthcare professionals in aerosol therapy and the progress in developing aerosols for systemic drug delivery, gene therapy and vaccines (thus discussing the challenges of the future). The availability of different kinds of inhalation devices can be confusing both for healthcare professionals and patients. For this reason, expertise and good communication skills are needed to teach the patient correctly and to guarantee that any single device is used properly. The course ended with clinical case discussions in adult and paediatric settings. It was a great opportunity for us to discuss practical problems with the experts in the field.

Finally, I would like to thank the faculty members and organisers for this interesting course, which gave me the opportunity to learn meaningful updates ready to be applied in daily clinical practice.

\section{Reference}

1. Boe J, Dennis JH, O'Driscoll BR. European Respiratory Society Guidelines on the use of nebulizers. Eur Respir J 2001; 18: 228-242. 